\title{
Strong acoustic plasmons in chemically doped graphene induced by a nearby metal surface
}

\author{
Vito Despoja,,${ }^{1,4,{ }^{*}}$ Dino Novko, ${ }^{1,4}$ Ivor Lončarić, ${ }^{2,4}$ Neven Golenić, ${ }^{1}$ Leonardo Marušić $\odot,{ }^{3}$ and Vyacheslav M. Silkin ${ }^{4,5,6}$ \\ ${ }^{1}$ Institute of Physics, Bijenička 46, HR-10000 Zagreb, Croatia \\ ${ }^{2}$ Ruđer Bošković Institute, Bijenička 54, HR-10000 Zagreb, Croatia \\ ${ }^{3}$ Maritime Department, University of Zadar, M. Pavlinovića 1, HR-23000 Zadar, Croatia \\ ${ }^{4}$ Donostia International Physics Center, P. Manuel de Lardizabal, 4, 20018 San Sebastián, Spain \\ ${ }^{5}$ Departamento de Fisica de Materiales and Centro Mixto CSIC-UPV/EHU, Facultad de Ciencias Químicas, Universidad del Pais Vasco \\ UPV/EHU, Apto. 1072, 20080 San Sebastián, Spain \\ ${ }^{6}$ IKERBASQUE, Basque Foundation for Science, 48013 Bilbao, Spain
}

(Received 17 July 2019; revised manuscript received 19 October 2019; published 4 November 2019)

\begin{abstract}
Recent theoretical considerations have demonstrated that free-standing graphene doped with alkali metals $\left(A \mathrm{C}_{x}\right)$ supports strong Dirac and weak acoustic plasmons. Here we show that when $A \mathrm{C}_{x}$ is deposited on a metal surface the effective Coulomb screening produced by the substrate can completely wash out these collective modes. However, even tiny detachment of the $A \mathrm{C}_{x}$ layer from the metal surface recovers the $A \mathrm{C}_{x}$ plasmonic properties. Especially the acoustic plasmon intensity is strongly enhanced in comparison to a free-standing case. We further provide the physical background of these intriguing phenomena. The studied systems consist of lithium- and cesium-doped graphene layers deposited on the $\operatorname{Ir}(111)$ surface.
\end{abstract}

DOI: 10.1103/PhysRevB.100.195401

\section{INTRODUCTION}

Ground-state crystal and electronic structures of graphene doped with alkali-metal atoms $\left(A \mathrm{C}_{x}, A=\mathrm{Li}, \mathrm{Na}, \mathrm{K}, \mathrm{Cs}\right)$ on different metallic surfaces, such as $\operatorname{Ir}(111), \mathrm{Cu}(111)$, and $\mathrm{Ni}(111)$, have been recently extensively studied in several experimental and/or theoretical papers [1-7]. The goal of these investigations was to achieve free-standing graphene (decoupled from the surface as much as possible) with the smallest moiré corrugation. Moreover, the above-mentioned experiments and further density functional theory (DFT) calculations [8] showed that graphene doped by alkali-metal atoms possesses an electronic band structure that could potentially support very interesting plasmonic properties. This is related to the fact that the alkali-metal atoms in a twodimensional (2D) superlattice become metallic and form a parabolic electronic $\sigma$ band that crosses the Fermi level [9]. Moreover, the alkali-metal atoms donate electrons to the graphene $\pi$ band, lifting the Fermi level to more than $1 \mathrm{eV}$ above the Dirac point [8]. As a result, coexistence of these partially occupied $2 \mathrm{D}$ energy bands in $A \mathrm{C}_{x}$ could support at least two electronic collective modes, where one is supposed to be a very strong Dirac plasmon (DP) [10,11].

The main goal of this paper is to emphasize the plasmonic effects existing in free-standing graphene doped by alkali metals. Recent theoretical investigations $[12,13]$ have already reported on a strong DP and a weak acoustic plasmon (AP) $[14,15]$ in $A \mathrm{C}_{x}$. However, here we focus on a much more realistic situation where doped graphene is deposited on metal surfaces [16-21]. Especially we aim to explore how the vicinity of the metal surface can be used to modify the DP and AP

\footnotetext{
*vito@phy.hr
}

dispersion relations and intensities. Investigation of graphenemetal heterostructures is actually of both fundamental [22] and practical $[23,24]$ importance. For instance, in the field of graphene-based biosensing [24] the graphene-metal contacts are unavoidable and thus deciphering the microscopic screening mechanisms in these structures is of great value.

In this paper we investigate the low-energy collective electronic excitations (2D plasmons) in lithium- and cesiumdoped graphene (i.e., $\mathrm{LiC}_{2}$ and $\mathrm{CsC}_{8}$ ) deposited on the $\operatorname{Ir}(111)$ surface. Special attention is paid to explore how the vicinity of the $\operatorname{Ir}(111)$ surface modifies the DP properties and enhances the AP intensities. We show how free-standing doped graphene supports a very strong DP and an AP with intensity weaker by two orders of magnitude. When the $A \mathrm{C}_{x}$ layer is located at the equilibrium position at the $\operatorname{Ir}(111)$ surface, the strong metallic screening in the substrate destroys the corresponding plasmonic excitations such that the DP in $\mathrm{LiC}_{2}$ becomes a very weak acousticlike branch, while the $\mathrm{DP}$ in $\mathrm{CsC}_{8}$ almost completely disappears. However, a small upward vertical displacement of the $A \mathrm{C}_{x}$ layer from the equilibrium position recovers the low-energy plasmon modes. Moreover this gives birth to interesting plasmonic phenomena not present in free-standing doped graphene. For instance, at a vertical displacement $\Delta$ of $0.6-1.2 \AA$ a very strong AP branch appears in $\mathrm{LiC}_{2}$. At displacement $\Delta$ of 1.2-1.6 $\AA$ the $\mathrm{CsC}_{8} / \mathrm{Ir}(111)$ supports two intense AP modes. At finite momentum transfers $(Q>0.1$ a.u. $)$ the intensities of these APs (lying in the infrared frequency range, $\omega>1 \mathrm{eV}$ ) can be even two orders of magnitude stronger than the DP intensities in the free-standing $A \mathrm{C}_{x}$ layer. These very intriguing plasmonic phenomena can be of interest in many plasmonics applications [25-36].

The rest of the paper is organized as follows. In Sec. II A we present a theoretical model for the ground-state crystal 
and electronic structure of the $\mathrm{LiC}_{2} / \operatorname{Ir}(111)$ and $\mathrm{CsC}_{8} / \operatorname{Ir}(111)$ systems. In Sec. II B we describe the method for calculating the effective 2D dielectric function $\epsilon(\mathbf{Q}, \omega)$ of $A \mathrm{C}_{x} / \operatorname{Ir}(111)$. In Sec. III the results for the calculated energy-loss function (ELF) $-\operatorname{Im}\left[\epsilon^{-1}\right]$ and real part of the effective $2 \mathrm{D}$ dielectric function $\operatorname{Re}[\epsilon]$ in $A \mathrm{C}_{x} / \operatorname{Ir}(111)$ are presented. Finally, we provide the conclusions in Sec. IV. When not stated otherwise, atomic units are used, i.e., $e=\hbar=m_{e}=1$, where $e$ is the electronic charge, $\hbar$ is the reduced Plank constant, and $m_{e}$ is the mass of the electron.

\section{THEORETICAL MODEL}

\section{A. Ground-state calculation}

The studied systems consist of a graphene monolayer doped with alkali-metal atoms and deposited on the $\operatorname{Ir}(111)$ surface $\left[A C_{x} / \operatorname{Ir}(111)\right.$ composite], as shown in Fig. 1. The separation between the alkali-metal atom layer in $A \mathrm{C}_{x}$ and the topmost $\operatorname{Ir}$ atomic layer of $\operatorname{Ir}(111)$ is

$$
h=d_{\mathrm{Ir}-A}+\Delta,
$$

where $d_{\mathrm{Ir}-A}$ represents the equilibrium separation obtained from the DFT calculations and $\Delta$ represents the vertical displacement from the equilibrium position.

The crystal structure of the $\operatorname{Ir}(111)$ surface is modeled with five atomic layers. A graphene $2 \times 2$ superlattice is matched to a $\operatorname{Ir}(111) \sqrt{3} \times \sqrt{3}$ superlattice such that $4.8 \%$ strain is applied to $\operatorname{Ir}(111)$. The unit cell in the $z$ direction is set to $L=22 \AA$.

Ground-state electronic and structural optimization calculations were performed using the QUANTUM ESPRESSO package $[37,38]$. The core-electron interaction is approximated by the norm-conserving pseudopotentials [39]. In order to capture the long-range van der Waals ( $\mathrm{vdW}$ ) interaction between the $\operatorname{Ir}(111)$ surface and doped graphene layers we use the $\mathrm{vdW}$ exchange-correlation functional [40,41], in particular the vdW-DF-cx version $[42,43]$. The ground-state properties in $A \mathrm{C}_{x} / \operatorname{Ir}(111)$ composites are calculated by using the $9 \times 9 \times 1$ Monkhorst-Pack K-point mesh [44], and the plane-wave cutoff energy is chosen to be $60 \mathrm{Ry}$. The structural optimization calculations are performed until the maximum force on each atom is reduced below $0.002 \mathrm{eV} / \AA$.

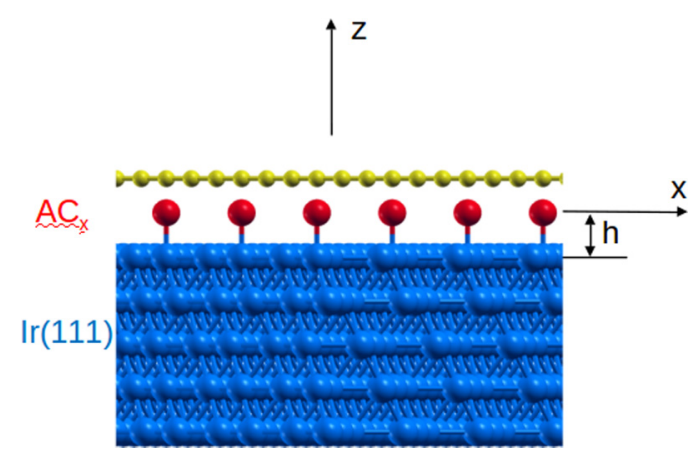

FIG. 1. Crystal structure of the alkali-metal atom doped graphene monolayer deposited on the $\operatorname{Ir}(111)$ surface [an $A \mathrm{C}_{x} / \operatorname{Ir}(111)$ composite].
TABLE I. The equilibrium separations (in $\AA$ ) between the topmost iridium atomic layer and the alkali-metal atom layer $d_{\mathrm{Ir}-A}$ as well as between the alkali-metal atom layer and the graphene monolayer $d_{A-\mathrm{C}}$ in the $A \mathrm{C}_{x} / \operatorname{Ir}(111)$ composites. The Fermi energies (in $\mathrm{eV}$ ) are relative to the graphene Dirac point.

\begin{tabular}{lcccc}
\hline \hline & $\mathrm{LiC}_{2}$ & $\mathrm{CsC}_{8}$ & $\mathrm{LiC}_{2} / \operatorname{Ir}(111)$ & $\mathrm{CsC}_{8} / \operatorname{Ir}(111)$ \\
\hline$d_{\mathrm{I}-\mathrm{A}}$ & & & 2.4 & 2.97 \\
$d_{A-\mathrm{C}}$ & 2.17 & 3.0 & 1.9 & 3.17 \\
$E_{F}$ & 1.78 & 1.24 & 1.61 & 0.95 \\
\hline \hline
\end{tabular}

The investigation of the plasmonic properties of the $A \mathrm{C}_{x} / \operatorname{Ir}(111)$ system is performed by treating the doped graphene $A C_{x}$ and the $\operatorname{Ir}(111)$ surfaces as nonoverlapping or chemically independent systems interacting by the longrange Coulomb potential. The Fermi levels in $\mathrm{LiC}_{2}$ and $\mathrm{CsC}_{8}$ samples, relative to the graphene Dirac point, are then frozen to their self-standing $(h \rightarrow \infty)$ values $E_{F}=1.78$ and $1.24 \mathrm{eV}$, respectively, regardless of the separation $h$. The ab initio calculations of the entire $A C_{x} / \operatorname{Ir}(111)$ system in equilibrium $\left(h=d_{\mathrm{Ir}-A}\right)$ give slightly lower values, $E_{F}=1.61$ and $0.95 \mathrm{eV}$. However, the influence of such a small change in the Fermi level on the $A \mathrm{C}_{x}$ plasmonic properties is negligible. The obtained vertical equilibrium separations between different atomic layers and Fermi levels (relative to the graphene Dirac point) in the $A \mathrm{C}_{x} / \operatorname{Ir}(111)$ composites are listed in Table I.

\section{B. Calculation of an effective 2D dielectric function}

Large equilibrium distance $d_{\mathrm{Ir}-A}$ results in small electronic overlap between the $A \mathrm{C}_{x}$ slab and the $\operatorname{Ir}(111)$ topmost layer which allows us to separate the calculation of the dynamically screened Coulomb interaction into two independent calculations, namely, computation of the $A \mathrm{C}_{x}$ noninteracting electron response function $\chi_{A C_{x}}^{0}$ and $\operatorname{Ir}(111)$ surface response function $D_{\mathrm{Ir}}$. Such approach considerably reduces the unitcell size and tremendously saves the computational time and memory requirements. This is indeed quite useful for studying the dynamical response in the $A \mathrm{C}_{x} / \operatorname{Ir}(111)$ composite when the $A \mathrm{C}_{x}-\operatorname{Ir}(111)$ distance is much larger than the equilibrium separation $h \gg d_{\mathrm{Ir}-A}$.

The ground-state electronic structures of $\mathrm{LiC}_{2}$ and $\mathrm{CsC}_{8}$ are first calculated using the equilibrium positions $d_{A-\mathrm{C}}$ as in the $A \mathrm{C}_{x} / \operatorname{Ir}(111)$ composite (see Table I) and using other parameters as described in Ref. [45]. The noninteracting electron response functions $\chi_{A C_{x}}^{0}$ are calculated using dense K-point grids, i.e., $201 \times 201 \times 1$ and $101 \times 101 \times 1 \mathrm{~K}$-point meshes for $\mathrm{LiC}_{2}$ and $\mathrm{CsC}_{8}$, respectively. The band summations in $\chi_{A C_{x}}^{0}$ are performed over 30 and 100 bands, for $\mathrm{LiC}_{2}$ and $\mathrm{CsC}_{8}$, respectively. In both cases the damping parameter $\eta=20 \mathrm{meV}$ is used. The calculation of the $\chi_{A C_{x}}^{0}$ is performed by fixing the Fermi energies $E_{F}$ and the separations $d_{A-\mathrm{C}}$ to the selfstanding $(h \rightarrow \infty)$ values, as stated in the two left columns of Table I. It should be noted here that for response function calculations the crystal local-field effects are included only in the perpendicular $(z)$ direction, i.e., the response functions are nonlocal only in the perpendicular direction and can be Fourier transformed as $\chi\left(z, z^{\prime}\right)=\frac{1}{L} \sum_{G_{z} G_{z}^{\prime}} e^{i G_{z} z-i G_{z}^{\prime} z^{\prime}} \chi_{G_{z} G_{z}^{\prime}}$ where $G_{z}$ and $G_{z}^{\prime}$ the are reciprocal-lattice vectors in the 
perpendicular direction. For $\chi_{A C_{x}}^{0}$ calculations we use a crystal local-field energy cutoff of $10 \mathrm{Ry}$, which corresponds to $23 G_{z}$ wave vectors. The dynamically screened Coulomb interaction can be calculated by solving the Dyson equation $w=v+$ $v \otimes \chi_{A C_{x}}^{0} \otimes w$, where $v=(2 \pi / Q) e^{-Q\left|z-z^{\prime}\right|}$ is bare Coulomb interaction [48] and $\otimes \equiv \int_{\infty}^{\infty} d z$.

In the vicinity of the polarizable $\operatorname{Ir}(111)$ surface the Coulomb interaction (e.g., the interaction between charge density fluctuations at $z, z^{\prime}>-h$ ) is mediated by the surface screened Coulomb interaction instead of the bare interaction $v$ :

$$
w_{\text {Ir }}=v+\frac{2 \pi}{Q} D_{\text {Ir }} e^{-2 Q h} e^{-Q\left(z+z^{\prime}\right)},
$$

where $D_{\mathrm{Ir}}=\frac{2 \pi}{Q}\left\langle e^{Q z_{1}}\left|\chi_{\mathrm{Ir}}\left(\mathbf{Q}, \omega, z_{1}, z_{2}\right)\right| e^{Q z_{2}}\right\rangle$ is the $\operatorname{Ir}(111)$ surface response function. The $\operatorname{Ir}(111)$ response function $\chi_{\text {Ir }}$ can be obtained by solving Dyson equation $\chi_{\mathrm{Ir}}=\chi_{\mathrm{Ir}}^{0}+\chi_{\mathrm{Ir}}^{0} \otimes v \otimes$ $\chi_{\mathrm{Ir}}$, where $\chi_{\mathrm{Ir}}^{0}$ represents the $\operatorname{Ir}(111)$ noninteracting electron response function. The ground-state electronic structure of the $\operatorname{Ir}(111)$ surface [45] is calculated using the $1 \times 1$ unit cell. The response function $\chi_{\mathrm{Ir}}^{0}$ is calculated using $101 \times 101 \times 1$ $\mathrm{K}$-point mesh, and the band summation is performed over 150 bands. The damping parameter $\eta=30 \mathrm{meV}$ is used. The crystal local-field energy cutoff of $10 \mathrm{Ry}$ is used, which equal to the $37 G_{z}$ wave vectors.

After the $A \mathrm{C}_{x}$ is deposited on the polarizable $\operatorname{Ir}(111)$ surface the bare Coulomb interaction $v$ has to be replaced by the surface screened Coulomb interaction $\left(v \rightarrow w_{\text {Ir }}\right)$, and the dynamically screened Coulomb interaction of the entire $A \mathrm{C}_{x} / \operatorname{Ir}(111)$ composite is calculated by solving the "screened" Dyson equation:

$$
w=w_{\text {Ir }}+w_{\text {Ir }} \otimes \chi_{A C_{x}}^{0} \otimes w .
$$

Finally, the effective 2D dielectric function can be defined as

$$
\epsilon^{-1}(\mathbf{Q}, \omega)=w\left(\mathbf{Q}, \omega, z=0, z^{\prime}=0\right) / v_{Q},
$$

where $v_{Q}=2 \pi / Q$. The energy-loss function directly probed in the reflection electron-energy-loss experiment [49] is then calculated as the imaginary part of $\epsilon^{-1}, S(\mathbf{Q}, \omega)=$ $-(1 / \pi) \operatorname{Im}\left[\epsilon^{-1}(\mathbf{Q}, \omega)\right]$.

\section{Long-wavelength limit $Q \approx 0$}

Due to the strong dispersivity of the dielectric response in the perpendicular $(z)$ direction it is not possible to neglect the crystal local-field effects in that direction and use the local 2D representation of the $A \mathrm{C}_{x}$ dielectric response. For example, the AP can be interpreted as an out-of-phase charge density oscillation in the alkali-metal atom $(A)$ and the graphene (C) plane, as has been theoretically demonstrated [12]. A similar plasmon has also been experimentally observed, propagating in the graphene $/ \mathrm{hBN} /$ metallic rod heterostructure [23]. Therefore, neglecting the crystal local-field effects for such layered heterostructures (which is equivalent to averaging in the $z$ direction) would remove the AP from the electronenergy-loss spectroscopy (EELS) spectrum. However, in the long-wavelength limit $(Q \rightarrow 0)$, the local 2D representation can be used even if the crystal local-field effects are neglected, by considering the $A \mathrm{C}_{x}$ layer as two $2 \mathrm{D}$ sheets, $A$ and $\mathrm{C}$, separated by $d_{A-C}$ and described by their local 2D dielectric functions $\epsilon_{A}(\omega)$ and $\epsilon_{\mathrm{C}}(\omega)$, respectively. Similar attempts have been successful in the experimental investigation [23]. In that case the effective 2D dielectric function of $A$-C sheets can be written as

$$
\epsilon_{2 \mathrm{D}}^{0}=\epsilon_{A} \frac{1-\alpha_{\mathrm{C}} \alpha_{A} e^{-2 Q d_{A-\mathrm{C}}}}{1+\alpha_{\mathrm{C}} e^{-2 Q d_{A-\mathrm{C}}}} .
$$

Here $\alpha_{i}=\frac{1-\epsilon_{i}}{\epsilon_{i}}$, the 2D dielectric function of a particular sheet is $\epsilon_{i}=1-\frac{2 \pi}{Q} \chi_{i}^{0}(Q, \omega)$, and the $2 \mathrm{D}$ response function is $\chi_{i}^{0}(Q, \omega)=L_{i} \chi_{i, G_{z}=0 G_{z}^{\prime}=0}^{0}(Q, \omega)$, where $i=\mathrm{C}$ or $A$. $\chi_{i, G_{z} G_{z}^{\prime}}^{0}(Q, \omega)$ are the ab initio noninteracting electron response functions of separated atomic $(i=A)$ and graphene $(i=\mathrm{C})$ layers, where $L_{i}$ represents the unit-cell constant in the perpendicular direction. The $\chi_{i}^{0}$ can also be obtained in a simpler model, such as the Dirac-cone approach [11] for $i=\mathrm{C}$ and one parabolic 2D band for $i=A$ [12]. This $A-\mathrm{C}$ sheet 2D model dielectric function does not include the dispersivity of the dynamical response perpendicular to the $\mathrm{C}$ and $A$ layers and it does not include intersystem $(\mathrm{C} \leftrightarrow A)$ electron-hole excitations. However, both effects are very small in the $Q \approx 0$ limit, and so Eq. (2) probably provides good AP and DP dispersion relations. In the presence of the iridium surface the effective dielectric function of the $A-C$ sheets becomes

$$
\epsilon_{2 \mathrm{D}}^{\mathrm{Ir}}=\frac{1-\alpha_{2 \mathrm{D}} D_{\mathrm{Ir}} e^{-2 Q h}}{1+\alpha_{2 \mathrm{D}}+D_{\mathrm{Ir}} e^{-2 Q h}+\alpha_{2 \mathrm{D}} D_{\mathrm{Ir}} e^{-2 Q h}},
$$

where $\alpha_{2 \mathrm{D}}=\frac{1-\epsilon_{2 \mathrm{D}}^{0}}{\epsilon_{2 \mathrm{D}}^{0}}$. For larger separations $h \gg d_{\mathrm{Ir}-A}$, which corresponds to the long-wavelength limit $\left(Q \approx \frac{1}{h}\right)$, the $\operatorname{Ir}(111)$ surface response function can be approximated as

$$
D_{\mathrm{Ir}}=\frac{1-\epsilon_{\mathrm{Ir}}}{1+\epsilon_{\mathrm{Ir}}}
$$

where $\epsilon_{\mathrm{Ir}}$ represents, for example, the bulk iridium macroscopic dielectric function. For smaller separations $h \approx d_{\mathrm{Ir}-A}$, the quantum nonlocal effects are important and the local description (4) becomes inaccurate. Therefore, the model 2D dielectric functions (2)-(4) possibly provide good DP and AP dispersion relations in the long-wavelength $Q \approx 0$ and $h \gg d_{\text {Ir-A }}$ limits, however it cannot be expected that they could give good AP and DP oscillatory strengths (e.g., their relative intensities), which is the main objective of this investigation.

\section{RESULTS AND DISCUSSION}

First we describe the modifications produced in the electronic excitation spectra of the $A C_{x}$ layer when it is brought from a free-standing $(\Delta \rightarrow \infty)$ to the equilibrium $(\Delta=0)$ distance at the $\operatorname{Ir}(111)$ slab. In addition, gradually increasing the separation $\Delta>0$, we present the corresponding spectra and explain the physical background of spectral alterations.

Figure 2(a) shows the ELF intensity in free-standing $(\Delta=\infty) \mathrm{LiC}_{2}$. As already reported in Ref. [12], in $\mathrm{LiC}_{2}$ the $\mathrm{Li}$ atoms donate electrons to the graphene $\pi^{*}$ band and additionally they form a parabolic $\sigma$ band which remains partially filled. Therefore, there are two bands crossing the Fermi level which result in two plasmons in $\mathrm{LiC}_{2}$, i.e., a strong DP and a weak AP [see Fig. 2(a)] as is the case in 

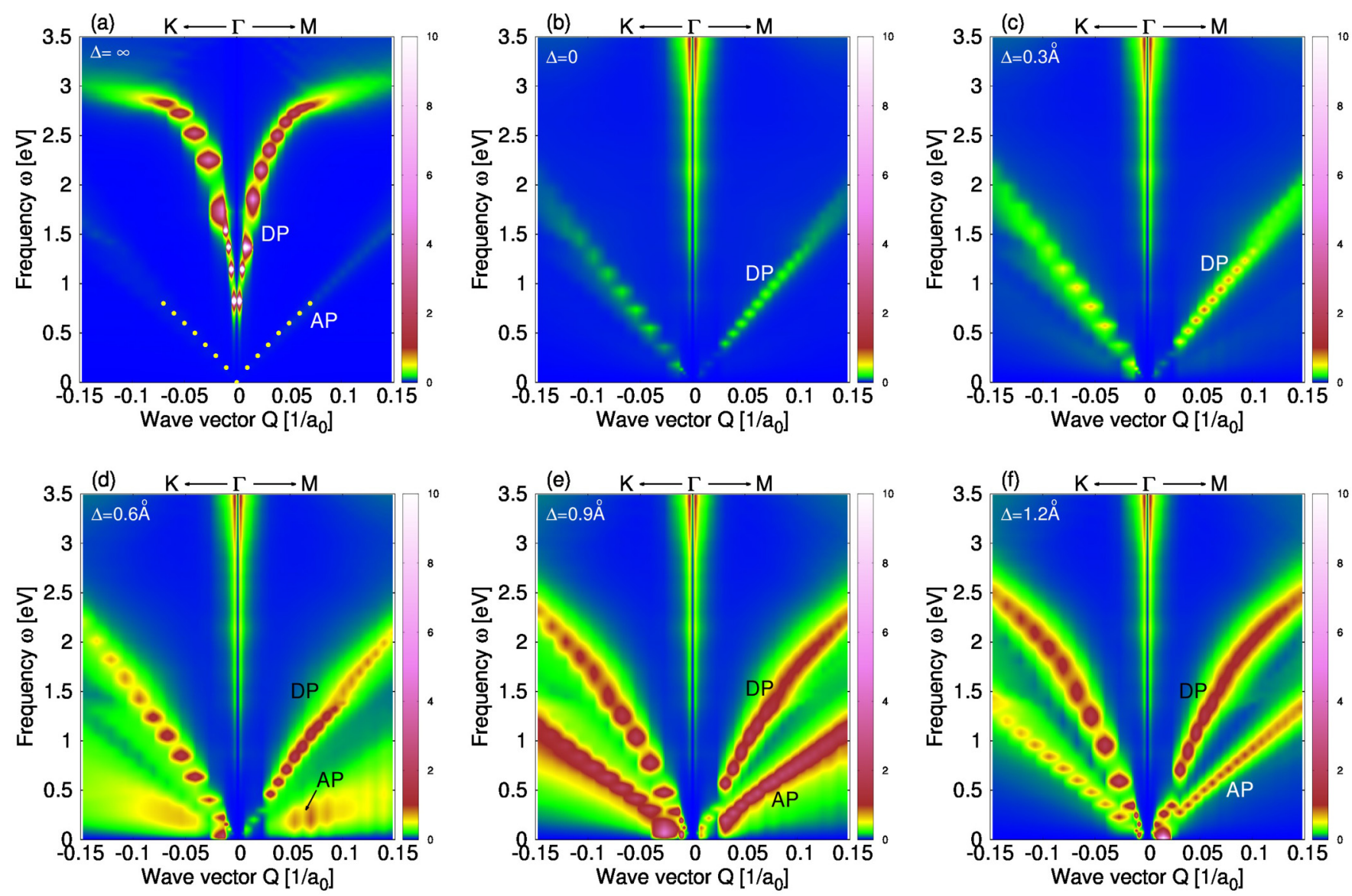

FIG. 2. The energy-loss-function intensity in $\mathrm{LiC}_{2} / \operatorname{Ir}(111)$ composite for (a) $\Delta=\infty$, (b) $\Delta=0$, (c) $\Delta=0.3 \AA$, (d) $\Delta=0.6 \AA$, (e) $\Delta=0.9 \AA$, and (f) $\Delta=1.2 \AA$. The unit for the wave vector is the inverse Bohr radius $\left(1 / a_{0}\right)$.

doped free-standing graphene $[14,15]$. Figure 2(b) shows the ELF intensities in $\mathrm{LiC}_{2}$ when it is located at the equilibrium distance $(\Delta=0)$ from the $\operatorname{Ir}(111)$ surface. It turns out that the metallic surface radically modifies the intensities of the electronic modes in $\mathrm{LiC}_{2}$. Namely, the DP signal becomes very weak and changes to a linearly dispersive mode due to efficient metallic screening in the substrate [50,51], while the AP mode disappears completely. Hence the strong metallic screening produced by the $\operatorname{Ir}(111)$ slab dramatically reduces the intensity of the electronic modes and pushes them toward lower energies. In order to clarify the situation, in Figs. 2(c)2(e) we show the ELF spectra of the $\mathrm{LiC}_{2} / \operatorname{Ir}(111)$ systems, where the separation between the $\mathrm{LiC}_{2}$ and $\operatorname{Ir}(111)$ films is gradually increased, from $\Delta=0.3$ to $1.2 \AA$.

Figure 2(c) shows that even small upward displacement with $\Delta=0.3 \AA$ from the equilibrium causes substantial increase of the DP intensity, while the dispersion remains linear. For $\Delta=0.6 \AA$ in the ELF shown in Fig. 2(d) the broad AP peak appears in the lower-energy region due to a less efficient dynamical screening in the substrate $[52,53]$ and the DP signal becomes notably stronger. Already for this separation the intensity of the AP peak is an order of magnitude stronger than the intensity of the $\mathrm{AP}$ in free-standing $\mathrm{LiC}_{2}$ [see Fig. 2(a)]. For $\Delta=0.9 \AA$ shown in Fig. 2(e) the dispersion relation of the DP bends (i.e., it is no longer linear) and its energy and intensity increase. The AP becomes a strong, sharp, and welldefined plasmon mode with intensity two orders of magnitude larger than in free-standing $\mathrm{LiC}_{2}$ and even stronger than the corresponding DP. For $\Delta=1.2 \AA$, shown in Fig. 2(f), the AP intensity reduces by an order of magnitude (in comparison with the $\Delta=0.9 \AA$ case) and the DP intensity continues to increase toward its free-standing $(\Delta=\infty)$ value. It is important to note that, since the metallic screening reduces the plasmon frequency, it pushes the DP out of the interband $\pi \rightarrow \pi^{*}$ continuum [54], which in turn reduces the Landau damping and increases the intensity of the plasmon compared to the free-standing case (e.g., compare the intensities in Figs. 2(e) and 2(f) with the intensities in Fig. 2(a) when $Q>0.1$ a.u.). Here we also have to mention the influence of the intraband $\pi^{*} \rightarrow \pi^{*}$ electron-hole excitations on the AP intensity in $\mathrm{LiC}_{2} / \operatorname{Ir}(111)$. In the self-standing sample $(\Delta \rightarrow$ $\infty)$ the linear AP follows the upper edge of the intraband $\pi^{*} \rightarrow \pi^{*}$ continuum $v_{F} Q$, where $v_{F}$ is the Fermi velocity in graphene [12]. However, considering that the vicinity of a metallic surface pushes the AP toward lower frequencies (i.e., it decreases the slope of the AP dispersion), for some smaller separations $\Delta$ it can be fully immersed in the intraband $\pi^{*} \rightarrow$ $\pi^{*}$ continuum. This causes the substantial damping of the $\mathrm{AP}$ in $\mathrm{LiC}_{2}$ deposited on the $\operatorname{Ir}(111)$ surface by the intraband $\pi^{*} \rightarrow \pi^{*}$ excitations, so in EELS it appears as a broad peak. This effect can be clearly seen in Fig. 2(d).

Also, we note that for the shown momentum-transfer interval the ELF intensities are almost isotropic, i.e., $S\left(\mathbf{Q}_{\Gamma \mathrm{M}}, \omega\right) \approx$ $S\left(\mathbf{Q}_{\Gamma \mathrm{K}}, \omega\right)$. 

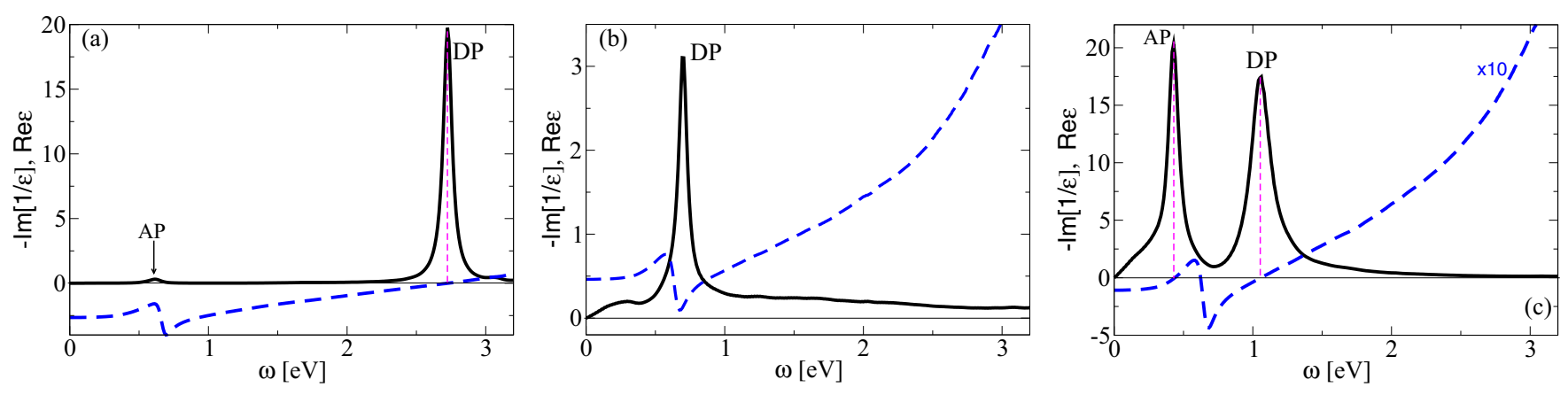

FIG. 3. The energy-loss function (black solid) and $\operatorname{Re}[\epsilon]$ (blue dashed) in $\mathrm{LiC}_{2} / \operatorname{Ir}(111)$ composite for (a) $\Delta=\infty$, (b) $\Delta=0$, and (c) $\Delta=$ $0.9 \AA$. The momentum-transfer vector with magnitude $Q=0.054$ a.u. is chosen to be in the $\Gamma$-M direction.

The panels in Fig. 2 clearly show that when the $\mathrm{LiC}_{2}$ $\operatorname{Ir}(111)$ distance is out of equilibrium with $\Delta>0$ the $\operatorname{Ir}(111)$ surface induces the strong AP but only for small intervals of separation $0.5<\Delta<1.0 \AA$. In order to understand such behavior, in Fig. 3 we show the real part of the effective 2D dielectric function (blue dashed lines) for three characteristic separations (a) $\Delta=\infty$, (b) $\Delta=0$, and (c) $\Delta=0.9 \AA$ and for $Q=0.054$ a.u. in the $\Gamma$-M direction. The corresponding ELFs are shown by black solid lines. It can be seen that in all the cases the $\operatorname{Re}[\epsilon]$ contains a "kink" structure which, depending on the $\Delta$ magnitude, shifts up and down as well as crosses zero at different frequencies. For $\Delta=\infty$ the kink, where the weak AP appears, is entirely below zero. At the higher frequencies, i.e., after the kink, the $\operatorname{Re}[\epsilon]$ increases and crosses the zero where the strong DP appears, classifying the DP as a welldefined collective electronic mode. On the other hand, when $\Delta=0$ the kink locates entirely above zero and $\operatorname{Re}[\epsilon]$ does not cross zero at all. This results in the appearance of the weak DP peak around the dip in $\operatorname{Re}[\epsilon]$. Considering that when $\Delta=\infty$ changes to $\Delta=0$ the kink in $\operatorname{Re}[\epsilon]$ transforms from entirely below to entirely above zero, there should be some $\Delta$ interval when the kink crosses zero. As can been seen in Fig. 3(c) this situation occurs, e.g., when $\Delta=0.9 \AA$, resulting in the appearance of both the AP and the DP peaks. In this case both plasmons can be classified as strong well-defined collective modes, as is clear from Fig. 2(e). For slightly larger separation, i.e., $\Delta>1.0$, the kink falls entirely below zero and the AP signal quickly weakens. However, $\operatorname{Re}[\epsilon]$ continues to cross zero at larger frequencies where the strong DP appears, as seen in Fig. 2(f).

In Fig. 4(a) we show the ELF intensity of the free-standing $(\Delta=\infty) \mathrm{CsC}_{8}$ layer. In this system the graphene $\pi^{*}$ band crosses the Fermi level twice, which together with the partially filled parabolic $\operatorname{Cs}(\sigma)$ band [13] is sufficient for the appearance of the three plasmons, i.e., the strong DP and the two weak acoustic plasmons, which we dub $\mathrm{AP}_{1}$ and $\mathrm{AP}_{2}$. This nomenclature for acoustic plasmons is merely a formality, since their dispersion over the entire momentumtransfer range cannot be necessarily linear [15]. Figure 4(b) shows the ELF intensity in $\mathrm{CsC}_{8}$ when it is at equilibrium distance $(\Delta=0)$ from the $\operatorname{Ir}(111)$ surface. The strong metallic screening extremely weakens the oscillatory strengths of the electronic modes such that the $\mathrm{DP}$ and $\mathrm{AP}_{2}$ become weak and barely visible modes, while $\mathrm{AP}_{1}$ disappears. Figures 4(c)-4(e) show the ELF intensities in $\mathrm{CsC}_{8}$ when the separation between
$\mathrm{CsC}_{8}$ and the $\operatorname{Ir}(111)$ surface gradually increases, from $\Delta=$ 0.4 to $1.6 \AA$. For the $\Delta=0.4-\AA$ case presented in Fig. 4 (c) the ELF intensity slightly increases. However, at $\Delta=0.8 \AA$ the enhancement of the ELF intensity in Fig. 4(d) is already significant and a new $\mathrm{AP}_{1}$ plasmon appears. It can be noticed that $\mathrm{AP}_{1}$ and $\mathrm{AP}_{2}$ have square-root dispersions, which is especially visible in the $\Gamma$-M direction. In the $\Gamma-\mathrm{K}$ direction, $\mathrm{AP}_{1}$ is still a very broad emerging mode. For $\Delta=1.2 \AA$ in the ELF shown in Fig. 4(e) the strong $\mathrm{AP}_{1}$ and $\mathrm{AP}_{2}$ peaks signal well-defined plasmon modes. In the $\Gamma-\mathrm{K}$ direction $\mathrm{AP}_{1}$ and $\mathrm{AP}_{2}$ have well-separated dispersion relations, while in the $\Gamma$-M direction and for the larger momentum transfers $Q>$ 0.1 a.u. these modes are degenerate. An interesting feature occurs in long-wavelength limit $Q \approx 0$, especially in the $\Gamma$-M direction, where $\mathrm{AP}_{2}$ and the DP start as a one plasmon branch and then at $Q \approx 0.025$ a.u. they bifurcate, i.e., the $\mathrm{DP}$ continues as a square-root branch and $\mathrm{AP}_{2}$ continues as a linear branch. For $\Delta=1.6 \AA$ shown in Fig. $4(\mathrm{f}) \mathrm{AP}_{1}$ is already notably weakened; $\mathrm{AP}_{2}$ is still a very strong mode, especially for larger momentum transfers $Q>0.1$ a.u. in both the $\Gamma-\mathrm{M}$ and $\Gamma-\mathrm{K}$ directions; while the DP continues to strengthen. The observable bifurcation into $\mathrm{AP}_{2}$ and $\mathrm{DP}$ branches is still present, in both directions for $Q \approx 0.05$ a.u. As already discussed, in the $\mathrm{LiC}_{2}$ case, for $\Delta>2 \AA \mathrm{AP}_{1}$ and $\mathrm{AP}_{2}$ suddenly weaken and the DP becomes gradually stronger until its intensity reaches the freestanding $(\Delta=\infty)$ value.

In order to understand the appearance of the strong acoustic plasmons $\mathrm{AP}_{1}$ and $\mathrm{AP}_{2}$ in $\mathrm{CsC}_{8}$ for certain separations $\Delta$, in Fig. 5 we show the real parts (blue dashed lines) of the effective $2 \mathrm{D}$ dielectric function $(\operatorname{Re}[\epsilon])$ for three characteristic separations (a) $\Delta=\infty$, (b) $\Delta=0$, and (c) $\Delta=1.3 \AA$, and for $Q=0.094$ a.u in the $\Gamma-\mathrm{K}$ direction. The ELFs are shown by the black solid lines. Contrary to the $\mathrm{LiC}_{2}$ case, it can be seen that the $\operatorname{Re}[\epsilon]$ of $\mathrm{CsC}_{8}$ has two kinks which move up and down depending on the separation $\Delta$. This means that for certain separations $\Delta$ the $\operatorname{Re}[\epsilon]$ can cross zero three times, and, therefore, three well-defined plasmons appear. Figure 5(a) shows that both kinks are entirely below zero, which gives only weak plasmons. For larger frequencies, after the kink structures, $\operatorname{Re}[\epsilon]$ crosses zero, providing a relatively strong but quite broad DP. This is reasonable considering that for this momentum transfer the DP already enters the $\pi \rightarrow \pi^{*}$ interband continuum and is thus considerably Landau damped [54]. This effect can also be seen as a sudden decrease of DP intensity for $Q>0.05$ a.u. in Fig. 4(a). For $\Delta=0$ the kinks 

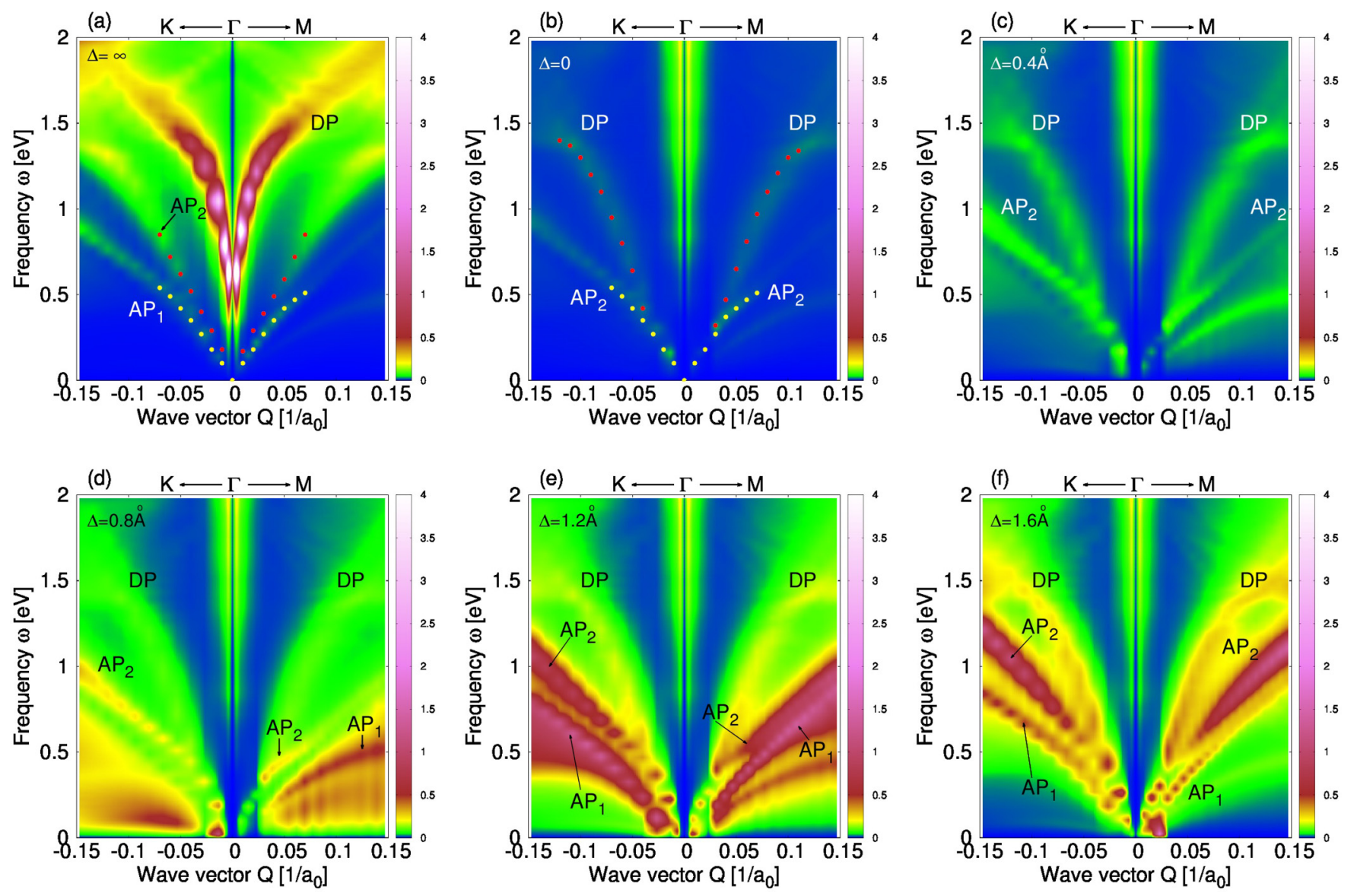

FIG. 4. The energy-loss-function intensity in $\mathrm{CsC}_{8} / \operatorname{Ir}(111)$ composite for (a) $\Delta=\infty$, (b) $\Delta=0$, (c) $\Delta=0.4 \AA$, (d) $\Delta=0.8 \AA$, (e) $\Delta=1.2 \AA$, and (f) $\Delta=1.6 \AA$. The wave-vector unit is inverse Bohr radius $\left(1 / a_{0}\right)$.

are entirely above zero and $\operatorname{Re}[\epsilon]$ does not cross zero at all. This results in only two very weak plasmons $\mathrm{AP}_{2}$ and the DP around which the dips of the kink structures appear. Mapping the conclusions from the $\mathrm{LiC}_{2}$ case, we expect a narrow $\Delta$ interval as well for $\mathrm{CsC}_{8}$ where at least one of the kinks crosses zero. It should also be noted that for this system there is no $\Delta$ for which both kinks would cross zero simultaneously. Figure 5(c) shows that for $\Delta=1.3 \AA$ the first kink crosses zero two times, which results in strong and well-defined plasmons $\mathrm{AP}_{1}$ and $\mathrm{AP}_{2}$. The second kink is still above zero, resulting in a weak DP just below the dip in the kink. This corresponds to the situation shown in Fig. 4(e) where the two strong plasmons, i.e., $\mathrm{AP}_{1}$ and $\mathrm{AP}_{2}$, and the weak $\mathrm{DP}$ bands are present. It is important to note here that for $\Delta=1.3 \AA$ [Fig. 5(c)] the intensities of $\mathrm{AP}_{1}$ and $\mathrm{AP}_{2}$ are an order of magnitude larger than $\mathrm{AP}_{1}$ and $\mathrm{AP}_{2}$ intensities and more than two times larger than the DP intensity in $\mathrm{CsC}_{8}$ for equilibrium separation $\Delta=0$ [Fig. 5(c)], at the same momentum transfer $Q$. For slightly larger $\Delta>2.0 \AA$ both kinks fall below zero and $\mathrm{AP}_{1}$ and $\mathrm{AP}_{2}$ quickly weaken, however $\mathrm{Re}[\epsilon]$ continues to cross zero for larger frequencies where the stronger DP is present (not shown).

These results generally show that for a narrow interval of displacements [i.e., $0.5 \AA \leqslant \Delta \leqslant 1.0 \AA$ for $\mathrm{LiC}_{2} / \operatorname{Ir}(111)$
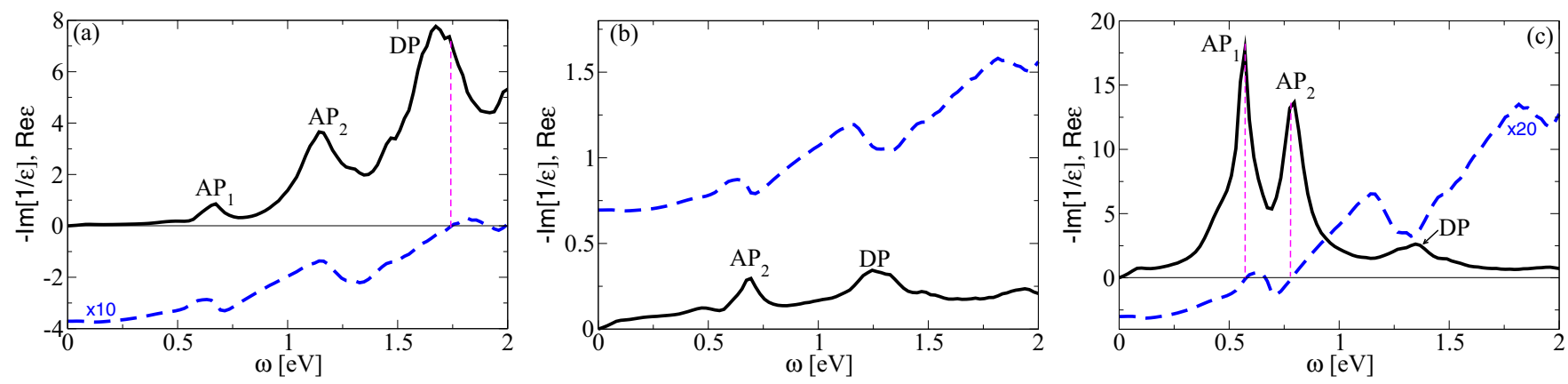

FIG. 5. The energy-loss-function (black solid) and $\operatorname{Re}[\epsilon]$ (blue dashed) in $\mathrm{CsC}_{8} / \operatorname{Ir}(111)$ composite for (a) $\Delta=\infty,(\mathrm{b}) \Delta=0$, and (c) $\Delta=$ $1.3 \AA$. The momentum-transfer vector with magnitude $Q=0.094$ a.u. is chosen to be in the $\Gamma$-K direction. 
and $1.0 \AA \leqslant \Delta \leqslant 1.5 \AA$ for $\left.\mathrm{CsC}_{8} / \operatorname{Ir}(111)\right]$ the $A \mathrm{C}_{x} / \operatorname{Ir}(111)$ composites support strong APs, which are for small momentum transfers (i.e., $Q<0.05$ a.u.) as strong as the DP in the free-standing cases. These APs persist as strong well-defined collective modes in a wide momentum-transfer interval (i.e., $0<Q<0.15$ a.u.) such that for larger momentum transfers $(Q>0.1$ a.u.) APs may be even three orders of magnitude stronger [see Figs. 2(e), 2(f), 4(e), and 4(f)] than the Landau damped DPs in free-standing systems [see Figs. 2(a) and 4(a)]. The metallic screening pushes the DP towards lower frequencies such that in the $\mathrm{LiC}_{2} / \operatorname{Ir}(111)$ composite the DP leaves the interband $\pi \rightarrow \pi^{*}$ continuum and becomes a sharp long-lived mode. Achieving these very interesting plasmonic features requires an artificial ejection of $A \mathrm{C}_{x} / \operatorname{Ir}(111)$ from the equilibrium distance $\Delta$ which might be experimentally feasible, for instance, by placing an inert single layer or a few layers of hexagonal boron nitride between graphene and the metal surface, as is actually the case in contemporary graphene-based devices $[22,23]$, or by replacing the alkali-metal atoms by larger intercalants, such as the $\mathrm{FeCl}_{3}$ molecules that act as electron acceptors lowering the Fermi energy below $-1 \mathrm{eV}$ [55]. Furthermore, the desirable plasmonic properties could be even achieved by changing the concept of the composite system. For example, instead of using metallic substrate one can use insulating substrates (e.g., $\mathrm{SiO}_{2}$ ), while the plasmonic properties in the $A \mathrm{C}_{x}$-substrate heterostructure could be manipulated by a dull metallic (e.g., aluminum) tip from above.

Finally, it should be recalled here that for all separations $\Delta$ the noninteracting electron response functions $\chi_{A C_{x}}^{0}$ are calculated such that we fix $d_{A-C}$ separations and Fermi energies $E_{F}$ to their self-standing values $(h \rightarrow \infty)$, as stated in the first two columns of Table I. Thus, the studied modifications of $A \mathrm{C}_{x}$ plasmonics are only due to long-range Coulomb interaction with the $\operatorname{Ir}(111)$ surface. The small modification of separation $d_{A-C}$ and Fermi energy $E_{F}$, consequently, with $\Delta$ has a minor impact on qualitative plasmonic properties, e.g., DP linearization and AP enhancement. The only expected quantitative effect is weak reduction of DP energy.

\section{CONCLUSIONS}

We have demonstrated how the 2D collective modes in chemically doped graphene are drastically modified in the presence of the $\operatorname{Ir}(111)$ metallic surface. For instance, a very strong Dirac plasmon present in $\mathrm{LiC}_{2}$ becomes two orders of magnitude weaker and linearly dispersive, while the acoustic plasmon disappears. Similar modifications were also observed in $\mathrm{CsC}_{8}$. Further, to understand these physical phenomena we gradually increased the graphene-surface separations $\Delta$ and analyzed the effective 2D dielectric function $\epsilon$. We have found that for the equilibrium separation $(\Delta=0)$ the strong metallic screening pushes the real part of the effective 2D dielectric function $(\operatorname{Re}[\epsilon])$ entirely above zero, which blocks the formation of well-defined 2D collective modes. However, for a narrow interval of out-of-equilibrium separations $(\Delta>0) \operatorname{Re}[\epsilon]$ crosses zero twice, providing two strong $2 \mathrm{D}$ plasmons. In particular, for $0.5 \AA \leqslant \Delta \leqslant 1.0 \AA$ the $\mathrm{LiC}_{2} / \operatorname{Ir}(111)$ composite supports strong well-defined Dirac and acoustic plasmons, while for $1.0 \AA \leqslant \Delta \leqslant 1.5 \AA$ the $\mathrm{CsC}_{8} / \operatorname{Ir}(111)$ composite contains two intense acoustic plasmons when the momentumtransfer magnitude is smaller than 0.15 a.u. All in all, we have shown that chemically doped graphene in the presence of a metallic surface could support even superior plasmonic features compared to the freestanding case. This manipulative plasmonic property can be used in many applications such as biosensing or in plasmon enhanced spectroscopic techniques.

\section{ACKNOWLEDGMENTS}

V.D. acknowledges support from the QuantiXLie Center of Excellence, a project cofinanced by the Croatian Government and European Union through the European Regional Development Fund within the Competitiveness and Cohesion Operational Programme (Grant No. KK.01.1.1.01.0004). V.D. is also grateful to Donostia International Physics Center (DIPC) for hospitality during various stages of this work. D.N. acknowledges financial support from the European Regional Development Fund for the "Center of Excellence for Advanced Materials and Sensing Devices" (Grant No. KK.01.1.1.01.0001). I.L. acknowledges support from the European Union through the European Regional Development Fund within the Competitiveness and Cohesion Operational Programme (Grant No. KK.01.1.1.06). V.M.S. acknowledges partial support from the Spanish Ministry of Economy and Competitiveness MINECO Grant No. FIS2016-76617-P and from Project No. PI2017-30 of the Departamento de Educación, Política Linguística y Cultura of the Basque government. Computational resources were provided by the DIPC Computing Center.
[1] P. Pervan, P. Lazić, M. Petrović, I. Š. Rakić, I. Pletikosić, M. Kralj, M. Milun, and T. Valla, Phys. Rev. B 92, 245415 (2015).

[2] J. Halle, N. Neel, and J. Kroger, Phys. Chem. C 120, 5067 (2016).

[3] M. Petrović, I. Šrut Rakić, S. Runte, C. Busse, J. T. Sadowski, P. Lazić, I. Pletikosić, Z.-H. Pan, M. Milun, P. Pervan, N. Atodiresei, R. Brako, D. Šokčević, T. Valla, T. Michely, and M. Kralj, Nat. Commun. 4, 2772 (2013).

[4] S. Tanaka, M. Petrović, K. Watanabe, P. Lazić, M. Kralj, T. Sugimoto, and Y. Matsumoto (unpublished).
[5] S. Schumacher, T. Wehling, P. Lazić, S. Runte, D. F. Forster, C. Busse, M. Petrović, M. Kralj, S. Blügel, N. Atodiresei, V. Caciuc, and T. Michely, Nano Lett. 13, 5013 (2013).

[6] B. Cook, A. Russakoff, and K. Varga, Appl. Phys. Lett. 106, 211601 (2015).

[7] M. Alattas and U. Schwingenschlogl, Sci. Rep. 6, 26753 (2016).

[8] D. Novko, Nano Lett. 17, 6991 (2017).

[9] I. Lončarić, Z. Rukelj, V. M. Silkin, and V. Despoja, npj 2D Mater. and Appl. 2, 33 (2018).

[10] B. Wunsch, T. Stauber, F. Sols, and F. Guinea, New J. Phys. 8, 318 (2006). 
[11] E. H. Hwang and S. Das Sarma, Phys. Rev. B 75, 205418 (2007).

[12] L. Marušić and V. Despoja, Phys. Rev. B 95, 201408(R) (2017).

[13] V. Despoja and L. Marušić, Phys. Rev. B 97, 205426 (2018).

[14] Y. Gao and Z. Yuan, Solid State Commun. 151, 1009 (2011).

[15] M. Pisarra, A. Sindona, P. Riccardi, V. M. Silkin, and J. M. Pitarke, New J. Phys. 16, 083003 (2014).

[16] H. Pfnür, T. Langer, J. Baringhaus, and C. Tegenkamp, J. Phys.: Condens. Matter 23, 112204 (2011).

[17] T. Langer, D. F. Forster, C. Busse, T. Michely, H. Pfnür, and C. Tegenkamp, New J. Phys. 13, 053006 (2011).

[18] A. Cupolillo, A. Politano, N. Ligato, D. M. C. Perez, G. Chiarello, and L. S. Caputi, Surf. Sci. 634, 76 (2015).

[19] A. Politano, H. K. Yu, D. Farías, and G. Chiarello, Phys. Rev B 97, 035414 (2018).

[20] A. Principi, E. van Loon, M. Polini, and M. I. Katsnelson, Phys. Rev. B 98, 035427 (2018).

[21] A. R. Echarri, J. D. Cox, and F. J. García de Abajo, Optica 6, 630 (2019).

[22] M. B. Lundeberg, Y. D. Gao, R. Asgari, C. Tan, B. Van Duppen, M. Autore, P. Alonso-Gonzalez, A. Woessner, K. Watanabe, T. Taniguchi, R. Hillenbrand, J. Hone, M. Polini, and F. H. L. Koppens, Science 357, 187 (2017).

[23] D. A. Iranzo, S. Nanot, E. J. C. Dias, I. Epstein, C. Peng, D. K. Efetov, M. B. Lundeberg, R. Parret, J. Osmond, J.-Y. Hong, J. Kong, D. R. Englund, N. M. R. Peres, and F. H. L. Koppens, Science 360, 291 (2018).

[24] D. Rodrigo, O. Limaj, D. Janner, D. Etezadi, F. J. García de Abajo, V. Pruneri, and H. Altug, Science 349, 165 (2015).

[25] M. Pumera, Materials Today 14, 308 (2011).

[26] E. Singh, M. Meyyappan, and H. S. Nalwa, ACS Appl. Mater. Interfaces 9, 34544 (2017).

[27] T. Mahmoudi, Y. Wang, and Y.-B. Hahn, Nano Energy 47, 51 (2018).

[28] Y. Miyoshi, Y. Fukazawa, Y. Amasaka, R. Reckmann, T. Yokoi, K. Ishida, K. Kawahara, H. Ago, and H. Maki, Nat. Commun. 9, 1279 (2018).

[29] T. Low and P. Avouris, ACS Nano 8, 1086 (2014).

[30] S. Huang, C. Song, G. Zhang, and H. Yan, Nanophotonics 6, 1191 (2017).

[31] M. Jablan, H. Buljan, and M. Soljačić, Phys. Rev. B 80, 245435 (2009)

[32] K. S. Novoselov, V. I. Falko, L. Colombo, P. R. Gellert, M. G, Schwab, and K. Kim, Nature (London) 490, 192 (2012).

[33] F. Schwierz, Proc. IEEE 101, 1567 (2013).

[34] F. Xia, Proc. IEEE 101, 1717 (2013).

[35] M. Jablan, M. Soljačić, and H. Buljan, Proc. IEEE 101, 1689 (2013).

[36] S. Xiao, X. Zhu, B.-H. Li, and N. A. Mortensen, Front. Phys. 11, 117801 (2016).
[37] P. Giannozzi, S. Baroni, N. Bonini, M. Calandra, R. Car, C. Cavazzoni, D. Ceresoli, G. L. Chiarotti, M. Cococcioni, I. Dabo et al., J. Phys.: Condens. Matter 21, 395502 (2009).

[38] P. Giannozzi, O. Andreussi, T. Brumme, O. Bunau, M. B. Nardelli, M. Calandra, R. Car, C. Cavazzoni, D. Ceresoli, M. Cococcioni et al., J. Phys.: Condens. Matter 29, 465901 (2017).

[39] N. Troullier and J. L. Martins, Phys. Rev. B 43, 1993 (1991).

[40] I. Lončarić and V. Despoja, Phys. Rev. B 90, 075414 (2014).

[41] C. Busse, P. Lazić, R. Djemour, J. Coraux, T. Gerber, N. Atodiresei, V. Caciuc, R. Brako, A. T. N'Diaye, S. Blügel, J. Zegenhagen, and T. Michely, Phys. Rev. Lett. 107, 036101 (2011).

[42] K. Berland, C. A. Arter, V. R. Cooper, K. lee, B. I. Lundqvist, E. Schroder, T. Thonhauser, and P. Hyldgaard, J. Chem. Phys. 140, 18A539 (2014).

[43] P. Hyldgaard, K. Berland, and E. Schroder, Phys. Rev. B 90, 075148 (2014).

[44] H. J. Monkhorst and J. D. Pack, Phys. Rev. B 13, 5188 (1976).

[45] DFT calculations of separate layers are performed in the same way as for the complete system, except the XC potential in $\mathrm{LiC}_{2}$ is approximated by the Perdew-Zunger local-density approximation [46] and that in $\mathrm{CsC}_{8}$ and the $\operatorname{Ir}(111)$ surface is approximated by the Perdew-Burke-Ernzerhof generalized gradient approximation [47]. We used $12 \times 12 \times 1,6 \times 6 \times$ 1 , and $12 \times 12 \times 1$ Monkhorst-Pack K-point mesh for $\mathrm{LiC}_{2}$, $\mathrm{CsC}_{8}$, and $\operatorname{Ir}(111)$, respectively. Supercells in the $z$ direction for $A \mathrm{C}_{x}$ are set to $12.3 \AA$ and those for the $\operatorname{Ir}(111)$ surface are set to $20.9 \AA$.

[46] J. P. Perdew and A. Zunger, Phys. Rev. B 23, 5048 (1981).

[47] J. P. Perdew, K. Burke, and M. Ernzerhof, Phys. Rev. Lett. 77, 3865 (1996).

[48] V. Despoja, Z. Rukelj, and L. Marušić, Phys. Rev B 94, 165446 (2016).

[49] V. U. Nazarov, New J. Phys. 17, 073018 (2015).

[50] J. M. Pitarke, V. U. Nazarov, V. M. Silkin, E. V. Chulkov, E. Zaremba, and P. M. Echenique, Phys. Rev. B 70, 205403 (2004).

[51] B. Diaconescu, K. Pohl, L. Vattuone, L. Savio, P. Hofmann, V. M. Silkin, J. M. Pitarke, E. V. Chulkov, P. M. Echenique, D. Farías, and M. Rocca, Nature (London) 448, 57 (2007).

[52] V. M. Silkin, A. García-Lekue, J. M. Pitarke, E. V. Chulkov, E. Zaremba, and P. M. Echenique, Europhys. Lett. 66, 260 (2004).

[53] L. Vattuone, M. Smerieri, T. Langer, C. Tegenkamp, H. Pfnür, V. M. Silkin, E. V. Chulkov, P. M. Echenique, and M. Rocca, Phys. Rev. Lett. 110, 127405 (2013).

[54] V. Despoja, D. Novko, K. Dekanić, M. Sunjić, and L. Marušić, Phys. Rev. B 87, 075447 (2013).

[55] F. J. Bezares, A. De Sanctis, J. R. M. Saavedra, A. Woessner, P. Alonso-Gonzalez, T. Amenabar, J. N. Chen, T. H. Bointon, S. Y. Dai, M. M. Fogler, D. N. Basov, R. Hillenbrand, M. F. Craciun, F. J. García de Abajo, S. Russo, and F. H. L. Koppens, Nano Lett. 17, 5908 (2017). 